\title{
Immortalization of human normal and NF1 neurofibroma Schwann cells
}

\author{
Hua Li', Lung-Ji Chang ${ }^{1}$, Debbie R Neubauer², David F Muir ${ }^{2,3,4}$ and Margaret R Wallace ${ }^{1,4,5}$
}

Neurofibromas, which are benign Schwann cell tumors, are the hallmark feature in the autosomal dominant condition neurofibromatosis 1 (NF1) and are associated with biallelic loss of NF1 gene function. There is a need for effective therapies for neurofibromas, particularly the larger, plexiform neurofibromas. Tissue culture is an important tool for research. However, it is difficult to derive enriched human Schwann cell cultures, and most enter replicative senescence after 6-10 passages, impeding cell-based research in NF1. Through exogenous expression of human telomerase reverse transcriptase and murine cyclin-dependent kinase (mCdk4), normal (NF1 wild-type), neurofibroma-derived Schwann cells heterozygous for NF1 mutation, and neurofibroma-derived Schwann cells homozygous for NF1 mutation were immortalized, including some matched samples from the same NF1 patient. Initial experiments employed retroviral vectors, while subsequent work utilized lentiviral vectors carrying these genes because of improved efficiency. Expression of both transgenes was required for immortalization. Molecular and immunohistochemical analysis indicated that these cell lines are of Schwann cell lineage and have a range of phenotypes, many of which are consistent with their primary cultures. This is the first report of immortalization and detailed characterization of multiple human NF1 normal nerve and neurofibroma-derived Schwann cell lines, which will be highly useful research tools to study NF1 and other Schwann tumor biology and conditions.

Laboratory Investigation (2016) 96, 1105-1115; doi:10.1038/labinvest.2016.88; published online 12 September 2016

Neurofibromatosis 1 (NF1) is a common autosomal dominant disease (birth incidence 1/2700 (ref. 1) with half of the patients bearing a de novo constitutional inactivating NF1 gene mutation. The vast majority of patients develop neurofibromas, benign Schwann cell tumors that can arise anywhere on the peripheral nervous system and throughout life. Adequate therapies to prevent or shrink these tumors do not yet exist. ${ }^{2}$ Although neurofibromas on the skin are small and mostly of cosmetic significance, those arising on larger nerves (termed plexiform) are larger and often cause morbidity. Plexiform neurofibromas can be fatal if they impinge on vital structures or become malignant. ${ }^{3}$

It is widely accepted that neurofibroma development in NF1 depends on a somatic NF1 mutation in a SC, rendering the cell deficient in NF1-encoded tumor suppressor protein neurofibromin function. ${ }^{4-6}$ Consistent with the size of the gene and high new mutation rate, there are no recurrent NF1 germline mutations with a frequency over $\sim 1-2 \%$ (except for whole-locus $\sim 1.5 \mathrm{Mb}$ deletions, in $\sim 7 \%$ of cases). ${ }^{7}$ Similarly, somatic mutations are widely variable (eg, Laycock-van Spyk et $a l^{8}$ ). Neurofibromin is known to be a key negative regulator of RAS signaling in SC, consistent with tumor suppressor activity, but has other (less-characterized) functions based on various studies (reviewed by Ratner and Miller ${ }^{9}$ ). Although the functional effects of NF1 mutations are actually quite complex and heterogeneous, ${ }^{7}$ reduced neurofibromin function, due to somatic NF1 mutation combined with germline mutation, is accepted as the pathogenic event initiating neurofibromas. Consistent with this, studies have generally failed to find additional and/or consistent somatic mutations at loci other than NF1, in neurofibroma Schwann cells, other than some tumors containing variably-sized deletions of chromosome 17 encompassing the non-germline-mutated NF1 allele. ${ }^{10-13}$

Cultivation of neurofibroma Schwann cells in the laboratory is important for cell, molecular and preclinical research, particularly because there are no naturally occuring animal models of NF1. In previous work, we and others established

\footnotetext{
Department of Molecular Genetics and Microbiology, University of Florida College of Medicine, Gainesville, FL, USA; ${ }^{2}$ Department of Pediatrics, Child Health Research Institute, University of Florida College of Medicine, Gainesville, FL, USA; ${ }^{3}$ Department of Neuroscience, University of Florida College of Medicine, Gainesville, FL, USA; ${ }^{4}$ University of Florida Health Cancer Center, University of Florida, Gainesville, FL, USA and ${ }^{5}$ University of Florida Genetics Institute, University of Florida, Gainesville, FL, USA Correspondence: Dr M Wallace, PhD, Department of Molecular Genetics and Microbiology, University of Florida College of Medicine, 1600 SW Archer Road, Gainesville, FL 32610-0266, USA.

E-mail: peggyw@ufl.edu

Received 6 February 2016; revised 3 July 2016; accepted 8 July 2016; published online 12 September 2016
} 
Schwann cell-enriched cultures from human NF1 tumors under conditions that favor the NF1-/- genotype. ${ }^{14-16}$ However, cultures derived from dermal and plexiform neurofibromas bear some residual fibroblasts, and nearly all enter replicative senescence, failing to divide beyond 6-9 passages. These cultures also require a prepared substratum such as laminin, and growth factor supplementation (eg, neuregulin 2). The lack of pure and easily cultured human SC (wild-type and from neurofibromas) has limited NF1 and related research. We sought to find an approach to overcome the limitations of these cultures, to provide more useful reagents for in vitro and in vivo studies.

There are several viruses/viral proteins that can immortalize mammalian cells in culture (provide capacity for unlimited replication ${ }^{17}$ ), including Epstein Barr virus, ${ }^{18}$ SV40 T antigen, ${ }^{19}$ adenoviruses $\mathrm{E} 1 \mathrm{~A}$ and $\mathrm{E} 1 \mathrm{~b},{ }^{20}$ and human papilloma virus 16 (E6 and E7 proteins). ${ }^{21}$ Although these processes are relatively reliable, they work with only certain cell types and require use of pathogenic agents. One group reported successful immortalization of normal human neonatal SCs by exogenously expressing both SV40 large $\mathrm{T}$ antigen and human reverse transcriptase component of telomerase (hTERT), ${ }^{22}$ but there have not been any further reports using this method for Schwann cells. Use of viral proteins may result in genetic instability or malignant transformation. There have been reports suggesting that exogenous expression of the reverse transcriptase component of telomerase (TERT) alone is also capable of immortalizing many human cell types (eg, fibroblasts, retinal pigmented epithelial cells, vascular endothelial cells, and mesothelial cells), but without subsequent genetic alterations. ${ }^{23-25}$ However, several other human cell types (eg, keratinocytes, mammary epithelial cells, bladder urothelial cells, and prostatic epithelial cells) transduced with TERT have subsequently been found to experience a telomerase-independent growth arrest that involves the $\mathrm{p} 16^{\text {Ink4a }} / \mathrm{pRB}$ pathway. ${ }^{26-28}$ Consistent with this, Darbro et al ${ }^{29}$ found hypomethylation of the CDKN2A promoter region (encodes $\mathrm{p} 16^{\text {Ink4a }}$ tumor suppressor protein) in telomerase-immortalized human keratinocytes co-cultured with feeder cells. This led to publication of an alternative method to immortalize human somatic cells by Ramirez et al, ${ }^{30}$ through expression of murine cyclin-dependent kinase (mCdk4) to overcome $\mathrm{p}^{\mathrm{Ink} 6^{\mathrm{In}}}$ mediated stress response, in combination with expression of the human telomerase catalytic subunit TERT (hTERT) to overcome replicative senescence. That team reported successful immortalization of human bronchial epithelial cells using retroviral delivery of $h T E R T$ and $m C D K 4$. Here we report use of the same strategy to generate immortal cell lines from human normal, NF1-patient heterozygous and neurofibromaderived NF1 two-hit Schwann cell cultures by initially retroviral, and subsequently lentiviral, vectors carrying the $h T E R T$ and $m C d k 4$ genes.

\section{MATERIALS AND METHODS Schwann Cell Primary Cultures}

Human normal (wild-type), NF1 (meeting diagnostic criteria $^{31}$ ) neurofibroma- and non-tumor nerve-derived SCs were isolated and cultured as previously reported ${ }^{16,32}$ with conditions for neurofibroma cultures favoring the enrichment of NFl - / - cells. These primary Schwann-enriched cultures require neuregulin (glial growth factor 2), and a laminin substratum. Pathology reports of the tumors indicated typical neurofibromas. These primary Schwann-enriched cultures require neuregulin (glial growth factor 2), and a laminin substratum. Nomenclature used for cell cultures and lines: $\mathrm{pNF}=$ plexiform neurofibroma SC from NF1 patient; $\mathrm{pnNF}=$ peripheral nerve SC from NF1 person from non-tumor nerve (heterozygous); $\mathrm{pn}=$ peripheral nerve SC from unaffected person.

\section{Transduction Using mCdk4 and hTERT Retroviruses}

Virus-producing cells expressing the respective transgenes PA317/hTERT and PA317/mCdk4 were kindly provided by Dr Woodring Wright, Univ of Texas Southwestern. ${ }^{30}$ These cells were cultured, and the virus-containing culture supernatant $(1: 100)$ in polybrene $(4 \mu \mathrm{g} / \mathrm{ml})$ was used to transduce adherent Schwann cells. pNF95.11b plexiform SC were first transduced with retro- $m C d k 4$ and placed under selection $(150 \mu \mathrm{g} / \mathrm{ml}$ G418 for 10 days), with surviving cells subsequently transduced with retro- $h T E R T$-containing supernatant (1:100) and polybrene, and subjected to selection $(300 \mathrm{ng} / \mathrm{ml}$ puromycin for 3-5 days).

\section{Transduction by lenti-hTERT and Retro-mCdk4}

Lenti-hTERT was produced by cloning the full-length human telomerase reverse transcriptase cDNA in front of the elongation factor $1-\alpha$-promoter in the vector pTYcPPT, $^{33}$ whereas lenti- $m C d k 4$ was produced by cloning the murine $C d k 4$ cDNA into the same vector. Separately from the retroviral transduction mentioned above, pNF95.11b plexiform SCs were transduced with lenti-hTERT per published protocols. ${ }^{34}$ Because there are no selectable markers in the lentiviral vector, cells were passaged until well beyond the passage at which the original cultures senesced (9-21 for this study), and surviving cells were transduced with retro- $m C d k 4$, followed by G418 selection. For this study, cells were considered immortalized if able to be passaged more than 50 times, because (1) that was well beyond the capacity of the primary cultures, (2) it suggested potentially unlimited capacity for cell division, (3) it is consistent with the Hayflick limit of replicative senescence, ${ }^{17}$ and (4) it fulfilled our goal of creating cell lines capable of being expanded significantly to fulfill potential needs of many downstream users. Most cultures were not tested for cell division beyond p50, although one (ipn02.3) was taken to p83 with no signs of replicative senescence. Cell lines were designated with an ' $i$ ' to indicate they were transduced with the transgenes and successfully immortalized, (si to designate semiimmortalization for pnNF95.12b, which senesced at P21). 


\section{hTERT and mCdk4 Transduction Using Only Lentivirus Vectors}

Based on improved transduction efficiency, all other SC cultures were transduced using both lentiviruses simultaneously: 10000 cells were transduced with 10-20 multiplicity of infection of both viruses for $12 \mathrm{~h}$ with $10 \mu \mathrm{g} / \mathrm{ml}$ polybrene in the media. Cells were maintained on laminin and neuregulin until passaged beyond senescence of the original culture, and then were tested for the ability to survive and proliferate without laminin and/or neuregulin (along with cultures transfected with retrovirus or a combination).

\section{Reverse Transcription (RT)-PCR}

RT-PCR for $h T E R T$ and $m C d k 4$ was performed to confirm expression of these transgenes in lenti-transduced cells, to confirm transgene expression. Primers for $m C d k 4$ (murinespecific): F: 5'-CGGGACATCAAGGTCACCCTA-3', R: 5'-GA ACAATGCAGTTTGCATGAAGA-3' (159 bp product). Primers for hTERT: F: 5'-CACTGGCTGATGAGTGTGTAC-3', R: 5'-TTCACCCTCGAGGTGAGACGCT-3' (320 bp product). GAPDH: F: 5'-TCATCATCTCTGCCCCCTCTG-3', R: 5'-GC CTGCTTCACCACCTTCTTG-3' (439 bp). Telomerase is not expressed at RT-PCR detectable levels in unmodified cultured Schwann cells (Techangamsuwan $e t a b^{35}$ and this report), so any expression was deemed transgene-related. In addition, RT-PCR was done to examine the NF1 transcript for inclusion of the $63 \mathrm{bp}$ alternative exon '23a' (traditional number in earlier literature, corresponds to number 31 in NG_009018.1) by PCR across this cDNA region using primers 5'-TCAACT TCGAAGTGTGTGCCAGTG-3' (XU7) and 5'-CACCATTGAT TTGACCAGTTTTG-3' (PTTS3-28R), followed by electrophoresis on a native polyacrylamide gel for ethidiumbromide visualization of relative amounts of transcript with (type II) and without exon 23a (type I).

\section{NF1 Mutation Analyses}

To assess the purity of the immortalized cultures, DNA analysis of the NF1 gene was performed to confirm the presence of the germline and somatic mutations in all but the wild-type lines (from normal human nerves). This was a twopart approach, the first based on established methods for PCR amplification of NF1 exons ${ }^{36}$ or cDNA fragments ${ }^{37}$ followed by mutation-specific restriction digest/polyacrylamide electrophoresis or by sequencing of the PCR products (BigDye 3.0 kit, Applied Biosystems/LifeTechnologies, Foster City, CA, USA). Sequence data were analyzed with Sequencher software (Genecodes, Ann Arbor, MI, USA). The second approach yielded the remaining mutations through highthroughput DNA sequencing strategies applied to the primary SC cultures. ${ }^{13}$

\section{Karyotype}

The Oregon Health Sciences University Cytogenetics laboratory provided standard G-banded karyotypes on the cell lines, characterizing 20 cells when possible. In brief, cells were harvested after $4 \mathrm{~h}$ of colcemid treatment to arrest in metaphase, incubated in a hypotonic solution $(0.075 \mathrm{M}$ $\mathrm{KCL}, 5 \%$ fetal bovine serum) for $10 \mathrm{~min}$, then fixed in 3:1 methanol:acetic acid. The cell suspension was dropped onto alcohol-cleaned microscope slides which were then baked at $90{ }^{\circ} \mathrm{C}$ for $20 \mathrm{~min}$. After cooling, the slides were trypsinized for $45 \mathrm{~s}$, stained with Wright stain for $80 \mathrm{~s}$, rinsed with distilled water and dried. Chromosomes were imaged using bright field microscopy and analyzed using Cytovision software (Applied Imaging, San Jose, CA, USA).

\section{Cell Authentication}

Single-cell authentication was performed on select lines by the Genetic Resources Core Facility at the Johns Hopkins University, using STR genotype profiling of immortalized cells and corresponding primary cultures (PowerPlex 18D system from Promega, Madison, WI, USA).

\section{Contact Inhibition}

Normal SC are contact inhibited in culture, meaning that they form a monolayer and stop dividing at confluence. In this assay, cells were plated as usual, such that the next day (day 1) had a density of $30-50 \%$. Each day for 5 days, photographs were taken to document the increasing density, morphology, and whether the cells were showing the ability to grow on top of each other (cell bodies, not just processes). The immortalized cultures were evaluated for whether, at confluence, the cells continued to divide and piled on top of each other, features of transformed cells. The latter phenotype was defined as lack of contact inhibition.

Immunocytochemistry for S100B and Proliferation Rate Immunocytochemistry was performed in eight-well chamber slides to stain cells for the SC marker S100B, and for BrdU incorporation to measure proliferation rate, using methods we reported previously and are briefly outlined here. ${ }^{16}$ For proliferation, cultures were assayed at passages where they showed optimal expansion, with the assay beginning at 50\% confluence. BrdU was added for $16 \mathrm{~h}$, following by fixation and immunostaining with anti-BrdU. The percentage of positive nuclei out of total nuclei in three different fields was calculated. S100B-positive cells were counted in triplicate (three fields) and averaged. To more simply represent the level of staining, a 1-4 scale based on relative S100B positivity was used for consistency with our publication describing several of the primary cultures, with ' 4 ' representing the most heavily immunopositive. ${ }^{16}$ These data are reported in Table 1.

\section{Doubling Time Assay}

For each cell line, $10^{4}, 3 \times 10^{4}$, and $10^{5}$ cells were seeded into 12-well tissue culture plates. At each of six time points $(24,48,72,96,120$, and $144 \mathrm{~h})$ viable cells in duplicate wells were counted (trypan blue/hemacytometer). Doubling times were calculated from these counts using the online program at www.doublingtime.com. 
Table 1 Characteristics of immortalized SC lines

\begin{tabular}{|c|c|c|c|c|c|c|c|c|c|c|c|c|c|c|c|c|}
\hline $\begin{array}{l}\text { Immortalized SC line } \\
\text { (transduced with lentiviral- } \\
\text { hTERT and-mCdk4, unless } \\
\text { otherwise specified) }\end{array}$ & Sex & $\begin{array}{l}\text { Germline NF1 } \\
\text { mutation }^{2}\end{array}$ & $\begin{array}{l}\text { Somatic } \\
\text { NF1 } \\
\text { mutation'2 }\end{array}$ & $\begin{array}{c}\text { Passage } \\
\text { number }(p) \text { at } \\
\text { which primary } \\
\text { culture } \\
\text { senesces }\end{array}$ & $\begin{array}{c}\text { Passage } \\
\text { number }(p) \\
\text { at } \\
\text { transduction }\end{array}$ & $\begin{array}{l}\text { Do cells } \\
\text { require } \\
\text { laminin, } \\
\text { neuregulin } \\
\text { (NRG)? }\end{array}$ & $\begin{array}{l}\text { Number } \\
\text { of } \\
\text { passages } \\
(p) \text { in } \\
\text { culture }\end{array}$ & $\begin{array}{c}\text { Immortal } \\
\text { S100B } \\
\text { category } \\
\text { (1 } \approx \text { all pos; } \\
4=\text { all neg.) }\end{array}$ & $\begin{array}{l}\text { Primary culture } \\
\text { S100B category } \\
\text { (1 } \approx \text { all pos; } \\
4=\text { all neg.) }\end{array}$ & $\begin{array}{l}\text { Cell morphology (may } \\
\text { vary with confluency) } \\
\text { (normal SC are spindle } \\
\text { shaped) }\end{array}$ & $\begin{array}{l}\text { Proliferation \% } \\
\text { BrdU 3/4th } \\
\text { confluent: primary, } \\
\text { immort (passage) }\end{array}$ & $\begin{array}{l}\text { Doubling } \\
\text { time in } \\
\text { hours } \\
\text { (passage) }\end{array}$ & $\begin{array}{l}\text { Do cells form } \\
\text { soft agar } \\
\text { colonies? (yes/ } \\
\text { no, passage) }\end{array}$ & $\begin{array}{l}\text { Cells display } \\
\text { contact } \\
\text { inhibition? } \\
\text { (primary, } \\
\text { immortalized) }\end{array}$ & $\begin{array}{l}\text { Cells form } \\
\text { tumor in } \\
\text { sciatic } \\
\text { xenograft? }\end{array}$ & $\begin{array}{l}\% \text { TUNEL } \\
\text { positive } \\
\text { (passage) }\end{array}$ \\
\hline $\begin{array}{l}\text { ipNF95.11b C/T plexiform } \\
\text { (transgenes from retrovirus) }\end{array}$ & M & c.1756delACTA & LOH 1 & p11 & $\begin{array}{l}\text { p8 lenti- } \\
\text { TERT, p11 } \\
\text { retro-mCdk44 }\end{array}$ & No, no & $>\mathrm{p} 50$ & 1 & 1 & Spindle & $32,21(\mathrm{p} 40)$ & 34.1 (р33) & No $\mathrm{p} 30$ & Yes, no & n.d. & 3.4 (p43) \\
\hline $\begin{array}{l}\text { ipNF95.11 b C same plexiform } \\
\text { (retrovirus-m } \mathrm{C} C \mathrm{k} 4 \mathrm{4} \text { ) }\end{array}$ & M & c.1756delACTA & LOH 1 & p11 & $\begin{array}{l}\text { p8 retro- } \\
\text { TERT, p20 } \\
\text { retro-mCdk4 }\end{array}$ & No, no & $>p 50$ & 1 & 1 & Fibroblastic and spindle & 32,18 (p53) & $30.7(\mathrm{p} 44)$ & No $\mathrm{p} 38$ & Yes, yes & n.d. & 9.7 (p54) \\
\hline ipNF95.6 plexiform & M & R816X & R2237X & p21 & p9 & No, no & $>$ p 50 & 4 & 3 & Fibroblastic and spindle & $63,47(p 47)$ & $45.0(\mathrm{p} 48)$ & No $\mathrm{p} 38$ & Yes, yes & Yes, small & $<1(p 51)$ \\
\hline ipNF05.5 plexiform & M & $\begin{array}{c}\text { c.3456_3457 } \\
\text { insA }\end{array}$ & LOH 1 & p7 & $\mathrm{p} 4$ & $\begin{array}{c}\text { No, no } \\
\text { (prefers } \\
\text { laminin; } \\
\text { grows faster } \\
\text { with NRG) }\end{array}$ & $>\mathrm{p} 50$ & 1 & 1 & Fibroblastic and spindle & $69,86(p 18)$ & $37.7(\mathrm{p} 15)$ & No $\mathrm{p} 22$ & Not done & n.d. & $0.3(\mathrm{p} 48)$ \\
\hline $\begin{array}{l}\text { ipNF05.5 (six-clone mix from } \\
\text { ipNF05.5) }\end{array}$ & M & $\begin{array}{c}\text { c.3456_3457 } \\
\text { insA }\end{array}$ & LOH 1 & p7 & $\begin{array}{l}\text { p4, clones } \\
\text { isolated at } \\
\text { p11 }\end{array}$ & $\begin{array}{l}\text { No, no } \\
\text { (prefers } \\
\text { laminin; } \\
\text { grows faster } \\
\text { with NRG) }\end{array}$ & $\begin{array}{c}\text { Slows } \\
\sim p 31, \\
+N R G= \\
>p 47\end{array}$ & 1 & 1 & Spindle & 69,82 & $40.3(\mathrm{p} 19)$ & No $\mathrm{p} 25$ & Yes, yes & n.d. & $6.3(\mathrm{p} 26)$ \\
\hline ipNF06.2A plexiform & $\mathrm{F}$ & G848W & Unknown & p15 & p5 & No, no & $>\mathrm{p} 50$ & 4 & 3 & Spindle & $49,32(\mathrm{p} 21)$ & $26.5(\mathrm{p} 20)$ & No p22 & Yes, no & No & $5.4(p 45)$ \\
\hline ipnNF95.11C heterozygous & M & c.1756delACTA & $n / a$ & p11 & p6 & No, no & $>\mathrm{p} 50$ & 4 & 1 & Epithelial/ variable & n.d., 50 (p20) & $38.8(\mathrm{p} 19)$ & No $\mathrm{p} 20$ & Partial, no & n.d. & $8.0(\mathrm{p} 30)$ \\
\hline ipn02.3 2ג normal & $\mathrm{F}$ & $n / a$ & $n / a$ & p10 & p5 & No, no & $>p 50$ & 3 & 1 & Spindle & $20,40(p 79)$ & $33.4(p 77)$ & No p75 & Yes, yes & No & $5.8(p 53)$ \\
\hline ipn02.8 normal & F & $n / a$ & $n / a$ & p10 & p3 & No, no & $>p 50$ & 3 & 1 & Fibroblastic & $61,15(p 21)$ & $25.6(p 21)$ & Yes p 20 & No, no & n.d. & $45.8(\mathrm{p} 19)$ \\
\hline ipn97.4 normal & M & $n / a$ & $n / a$ & p12 & p6 & No, no & $>\mathrm{p} 50$ & 1 & 1 & Epithelial & $49,33(\mathrm{p} 20)$ & $24.7(\mathrm{p} 18)$ & Yes p17 & Yes, no & n.d. & $44.0(\mathrm{p} 20)$ \\
\hline sipnNF95.12B heterozygous & $\mathrm{F}$ & L216P & n/a & p10 & p1 & Yes, yes & p21 & 4 & 1 & Spindle & n.d. & $82.4(\mathrm{p} 18)$ & No p17 & Yes, partial & n.d. & $11.3(\mathrm{p} 18)$ \\
\hline ipnNF09.4 heterozygous & M & $\begin{array}{c}\text { c.3456_3457 } \\
\text { insA }\end{array}$ & $n / a$ & p5 & p3 & Yes, yes & $>$ p47 & 1 & 1 & Fibroblastic and spindle & $50,19.7(\mathrm{p} 25)$ & $59.9(\mathrm{p} 18)$ & No 17 & Yes, yes & n.d. & $9.0(\mathrm{p} 46)$ \\
\hline ipNF00.6 plexiform & $\mathrm{F}$ & $\begin{array}{l}\text { gene deletion } \\
(>1 \mathrm{Mb})\end{array}$ & Unknown & p10 & p5 & Yes, yes & $>\mathrm{p} 50$ & 2 & 1 & Spindle & 65,27 (p21) & $51.4(\mathrm{p} 21)$ & No p19 & Yes, yes & No & n.d. \\
\hline ipNF03.3 plexiform & M & $\begin{array}{l}\text { c.4269 G>A } \\
\text { in-frame exon } \\
\text { skip }\end{array}$ & Unknown & p7 & $\mathrm{p} 4$ & No, no & $>\mathrm{p} 50$ & 1 & 2 & Fibroblastic & 20,67 (p20) & $39.2(\mathrm{p} 18)$ & Yes p17 & Yes, no & Yes & $44(\mathrm{p} 48)$ \\
\hline ipNF04.4 plexiform & $\mathrm{F}$ & R2237X & LOH 1 & p8 & p5 & No, no & $>\mathrm{p} 50$ & 1 & 2 & Epithelial & $22,41(p 18)$ & $26.6(\mathrm{p} 25)$ & Yes p24 & Yes, no & Yes & $40(\mathrm{p} 20)$ \\
\hline
\end{tabular}

LOH 1, loss of heterozygosity, somatic deletion; n/a, not applicable; n.d., not determined; p, passage; SC, Schwann cell. Reference seq.: NM_000267.3 and NP_000258.1. 


\section{Soft Agar Assay}

This measure of tumorigenicity (anchorage-independent growth) was performed as we described previously, using the glioblastoma cell line T98G as positive control. ${ }^{16}$

\section{TUNEL Staining For Apoptosis}

Apoptotic index of each cell line was determined using the Promega DeadEnd TUNEL system according to the manufacturer's instructions (Promega).

\section{Microsatellite Instability}

D5S346, D17S250, and BAT26, three established NCI microsatellite instability markers, ${ }^{38}$ were genotyped using standard PCR conditions (HotStar Taq polymerase, Qiagen, Valencia, CA, USA) followed by $15 \%$ native polyacrylamide gel electrophoresis to screen for de novo alleles in the cells' DNA compared with that of primary cultures or germline DNA. BAT26 PCR primers were redesigned to create a smaller amplimer (155 bp) for better gel resolution of individual alleles: 5'-GACTACTTTTGACTTCAGCCAGTA-3' and 5'-GCTTCTT CAGTATATGTCAATGAAAAC-3'. Further, one of the cell authentication markers used (PENTAD) also provided insight about microsatellite instability.

\section{Xenografts}

Taking advantage of occasional opportunities for a pilot test of tumorigenicity in vivo, we were able to test six cell lines by xenograft, placed subcutaneously or intraneurally into NODscid-IL2R $\gamma$ mice (Jackson Laboratories \#005557, Bar Harbor, Maine, USA) as we described previously, ${ }^{16}$ to detect whether visible tumors developed or not (microscopic analysis was not undertaken). In our experience, primary neurofibromaderived SC do not form subcutaneous masses. When xenografted into nerve, neurofibroma-derived SC usually survive and may proliferate, but do not tend to form a visible tumor mass (or they are small), unlike many cell lines derived from malignant peripheral nerve sheath tumor (MPNST) that create large tumors. ${ }^{16,39-41}$

\section{Results}

Primary Schwann cell cultures established from patient surgical donations were the primary material for this work. This included three cultures from non-tumor nerve of subjects with NF1, leading to matched heterozygous SC lines for two of the subjects whose NF1-null Schwann cells also immortalized (ipNF95.11b C and ipnNF95.11c; ipNF05.5 and ipnNF09.4). No primary cultures divided beyond p21, and most senesced in less than 10 population doublings. ${ }^{16}$

In the initial experiments transducing several cultures with retroviral vectors containing $h T E R T$ and $m C d k 4$, the vast majority of cells died during selection, indicating a poor transduction rate. Only one cell line resulted from retroviral transduction of plexiform neurofibroma-derived SC culture pNF95.11b: immortalized culture ipNF95.11b 'C' (via retro$m C d k 4$ first followed by retro-hTERT infection). We then tried transduction of a SC culture using a lentivirus carrying only the GFP gene as a label, and found $80 \%$ transduction efficiency (data not shown), leading to subsequent choice to use lentiviral vectors for all remaining immortalizations. During the transition, however, pNF95.11b immortalized cell line ' $\mathrm{C} / \mathrm{T}$ ' was also produced, by lenti- $h T E R T$ transduction (at p8) followed by retro-mCdk4 (at p20). Table 1 lists the immortalized lines and specific properties, including comparison with properties of the corresponding primary cultures.

Transduction with retro- or lenti- $h T E R T$ or $m C d k 4$ alone did not produce any immortalized SC lines except a $h T E R T$ only culture ( $h T E R T$-pn02.3), which expanded up to p38 before it stopped proliferating. The observation that SC required both transgenes for immortalization is consistent with literature indicating that some normal cell types require both proteins (hTERT and $\mathrm{mCdk} 4$ ) to overcome replicative senescence in culture. ${ }^{30} \mathrm{We}$ have not tested expansion beyond p50 except for the normal Schwann cell line ipn02.3 $2 \lambda$, which has divided to p83 with no signs of senescence, and has been utilized in studies by a number of other laboratories. ${ }^{42-46}$ The cultures were each immortalized as a population and studied as such, although we wanted to test whether single-cell cloning was possible. We were able to derive at least a few single-cell clones from the cell lines tested: ipNF05.5, ipNF95.6, ipNF04.4, and ipn02.3 2 $\lambda$. As a side experiment, six individual clones (NF1-null) that were cloned at passage 11 from ipNF05.5 were combined at passage 16 to compare to the original immortalized ipNF05.5. It was reassuring that both the population and mixed clones showed similar properties for most of the features in Table 1 and Supplementary Table S2, and also yielded similar results in a primary screen against a small drug library. ${ }^{47}$

For simultaneous double lentiviral transduction, cultures divided beyond p50, except that ipnNF09.4 was only taken to $\mathrm{p} 47$, but did not show signs of senscence at that point, and sipnNF95.12B which sensesced at P21. Cell authentication genotyping confirmed that the immortalized lines derived from their corresponding primary cultures (STR genotypes listed in Supplementary Table S1). All of the immortalized lines became laminin- and neuregulin-independent except for ipNF00.6, although two lines (ipNF05.5 and the six-clone mix of ipNF05.5) grew more robustly with both.

As shown in Figure 1a, the two lines derived from pNF95.11b appear to be pure or nearly pure two-hit cells, showing loss of heterozygosity with no evidence of the normal allele. DNA sequencing of ipNF95.6 showed that the somatic NF1 mutation (nonsense R2237X) is present in equal quantities with the germline allele (a nonsense mutation at another site, but wild type at the somatic mutation base) (Figure $1 \mathrm{~b}$ ), consistent with the majority of cells containing both the germline and somatic mutation, despite lack of S100B expression. This supports the conclusion that this line represents relatively pure immortalized 'two-hit' tumor SC rather than heterozygous cells. Four such plexiform cell lines were generated with both germline and somatic mutations 
confirmed (Table 1); in three others (ipNF06.2A, ipNF00.6, ipNF03.3) the somatic mutation was not identified despite indepth screening, so it is likely that these represent heterozygous SC that were by chance immortalized rather than the two-hit SC (or, the somatic mutation may be present, but such cells are at extremely low frequency). In the three heterozygous cell lines derived from NF1 patient nerve with no evidence of tumor involvement, the germline mutations were confirmed (ipnNF95.11C, ipnNF95.12B, and ipnNF09.4). In further genetics experiments, semiquantitative RT-PCR analysis of the NF1 type II alternatively spliced transcripts showed that at least half of the NF1 transcripts in the immortalized cell lines represented the type II isoform that includes exon '23a' (Figure 2a). This isoform is associated with reduced RAS GTPase-activating (GAP) activity, thus greater active RAS-GTP signaling. ${ }^{48-49}$ Some cell lines were closer to half type I and half type II, including the normal SC lines. Normal adult tissues typically show more
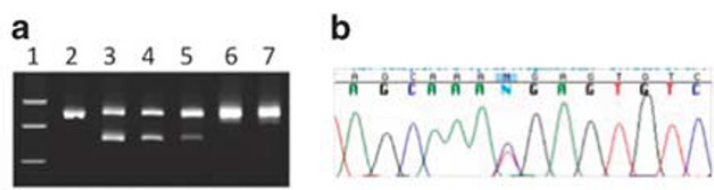

Figure 1 Molecular analysis of two immortalized plexiform SC lines. (a) Loss of heterozygosity analysis of pNF95.11b, using SNP marker rs964288 in the NF1 gene intron 41 (genotyped by Pacl digestion). Lane 1 contains a molecular weight ladder. Lane 2 shows an uncut PCR product for reference. Lane 3 shows Pacl digest of the PCR product from the patient's leukocyte (germline) DNA, showing heterozygous genotype. Lane 4 contains the Pacl digest from the primary tumor PCR product, showing slight loss of heterozygosity, consistent with tissue admixture. Lane 5 shows the genotype of the primary tumor SC culture (p8) showing some enrichment for the two-hit SC. Lane 6 shows that immortalized culture " $\mathrm{C} / \mathrm{T}$ " (p43) has no evidence of the wild-type allele despite overloading, as also seen in lane 7 for the " $\mathrm{C}$ " line (p41). (b) A sequencing chromatogram shows approximately equal quantities of the " $\mathrm{C}$ " germline allele and the somatic mutant "T" allele in SC line ipNF95.6 (p42) at CDNA position 6709 (NM_000267.3) (shown as "N" on chromatogram) encoding R2237X nonsense mutation. type I or equal levels of both. ${ }^{50}$ The immortalized lines tended to have the same isoform patterns as their original cultures, which in turn were consistent with isoform patterns in the primary tissue. ${ }^{51}$

Semi-quantitative RT-PCR data verified expression of both transgenes in all cell lines, including at multiple passage numbers (Figure 2). Vector copy number of one line (ipNF00.6) that slowed in proliferation rate at p27 was checked; that line had four lentiviral copies present and with relatively equal expression both at p8 and p27 (data not shown). Thus, the transgenes appear to be stably integrated and active in these lines, consistent with established lentivirus biology.

Most cell lines had at least some cells immunopositive for the Schwann cell marker S100B (Table 1, scores 1-3), consistent with being of Schwann lineage. This was true even for the cell lines whose morphology had gained some polygonal (epithelial-like) or fibroblastic morphology, in addition to spindle-like (typical of SC), where cells of different morphology stained similarly for S100B (Table 1). Most of the immortal lines were equally or somewhat less immunopositive for S100B compared with original untransfected cultures as illustrated in Figure 3 (pNF95.6 vs ipNF95.6, pNF95.11b vs ipNF95.11b showing similarity), regardless of apparent high purity of homozygous mutant cells. Among the minority showing less similarity were two heterozygous cell lines that lost S100B staining relative to primary culture, and in addition, ipNF03.3 and ipNF04.4 became fully S100B-positive after immortalization. Thus, while generally consistent relative to primary culture, the S100B phenotype was seen to vary as a result of the immortalization process, regardless of cell morphology change. Therefore, the $\mathrm{S} 100 \mathrm{~B}$ phenotype is not a robust marker for whether immortalized lines are of Schwann origin; instead, molecular work demonstrating that tumor-derived immortalized cells carry NF1 germline and somatic mutations is a better measure of whether such lines truly derive from the original tumor SC.
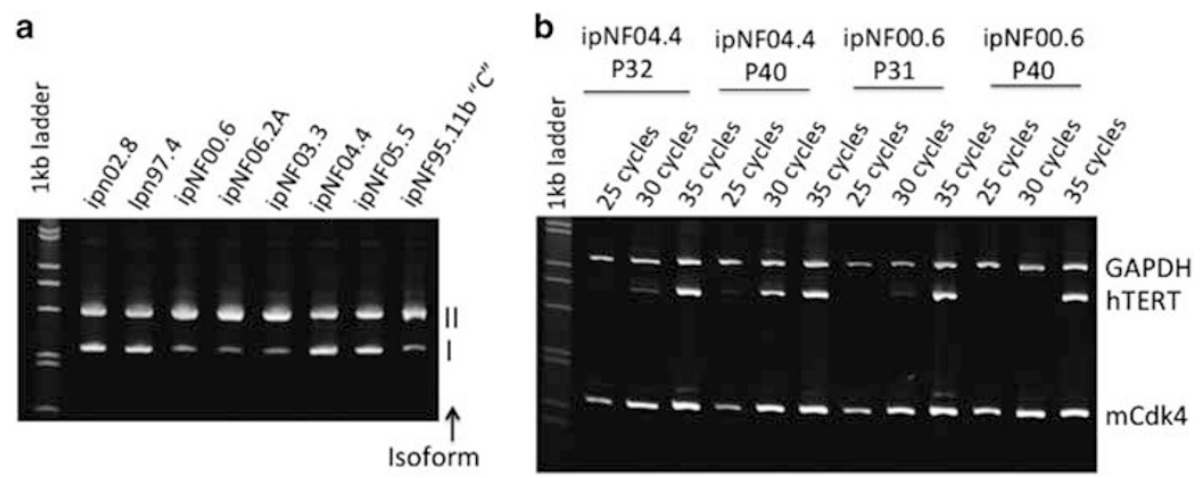

Figure 2 RT-PCR studies of isoform usage and transgene expression, visualized on polyacrylamide gels. (a) Cell lines exhibited inclusion of the NF1 alternatively spliced exon "23a" (type II) in half or more of transcripts. (b) A representative semi-quantitative RT-PCR analysis (sampled at 3 different cycles) confirms stable expression of both transgenes in the cell lines, at multiple passages. GAPDH is the loading control. 


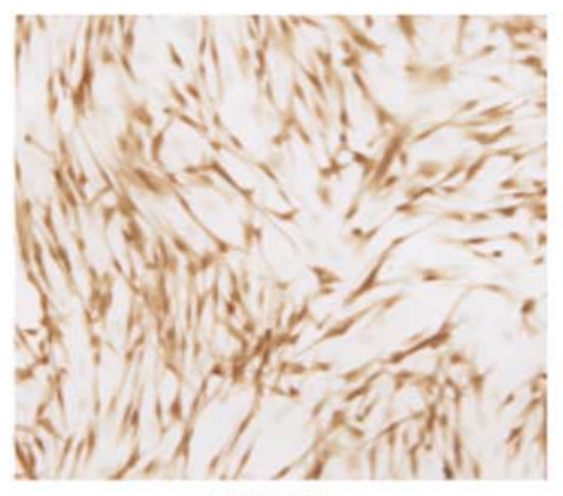

pNF95.11b

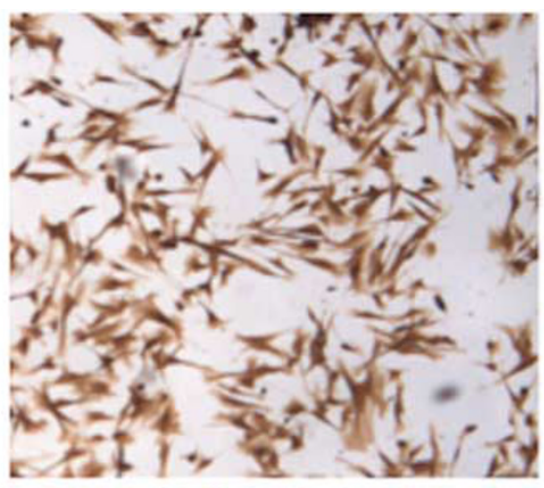

ipNF95.11b C

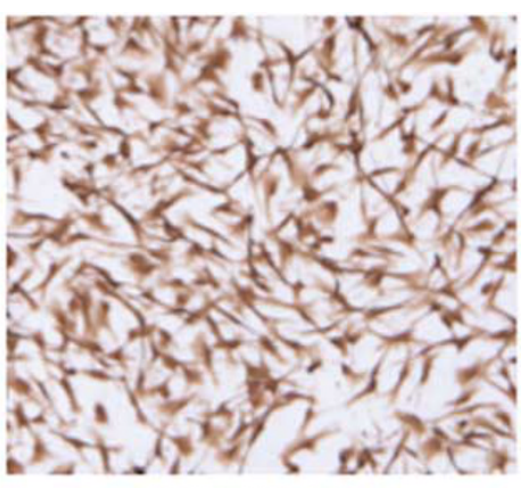

ipNF95.11b C/T

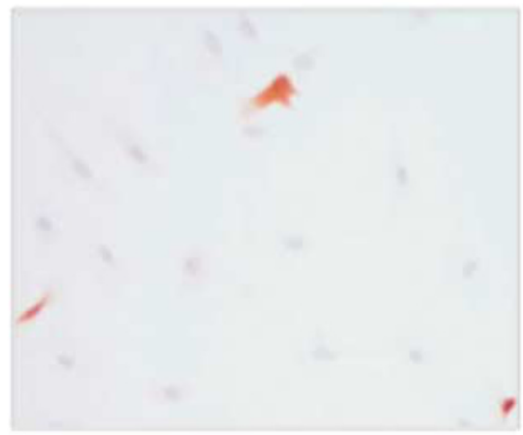

pNF95.6

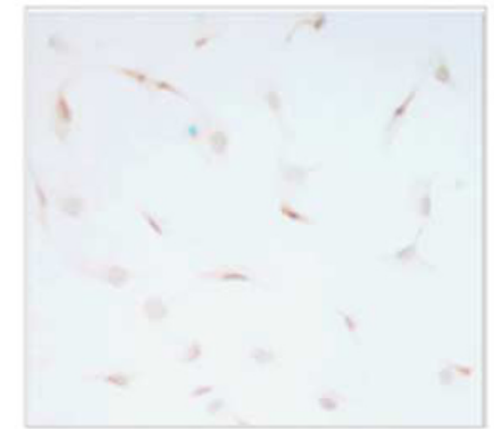

ipNF95.6

Figure 3 Examples of S100B immunostaining showing similar results for primary and immortalized cultures. Similar strong S100B staining is seen in pNF95.11b original culture (p9) and corresponding immortalized cultures ipNF95.11b " $\mathrm{C}$ " (p28) and " $\mathrm{C} / \mathrm{T}^{\prime}$ (p28). On the bottom panel, a small minority of cells in the pNF95.6 primary culture (p9) are S100-positive, while no staining is detected in the corresponding immortalized culture ipNF95.6 (p42).

There was heterogeneity among the cell lines with respect to most other measures, reflecting similar wide range in properties measured in the primary cultures $\left({ }^{16}\right.$ and Table 1$)$ and reported in many human tumor cell lines even with the same histopathological diagnosis. For example, studies showed that some of the immortalized lines had higher proliferation indices than primary cultures (example in Figure 4); while some were lower (some statistically significant, some not), overall representing heterogeneity similar to that reported among primary human SC cultures. ${ }^{15,16}$ Similarly, the percentage of apoptotic cells in the immortalize lines ranged from virtually none to $44 \%$, and doubling times also had a wide range (24-82 h). But there were no consistent patterns between immortalized phenotypes. For example, some SC lines had a relatively higher prolifertion rate and lower apoptotic rate, while others were lower/lower, or lower/higher, and these observations did not correlate with the cells being homozygous NF1 mutant, or other properties in any clear fashion. No primary cultures were able to grow in soft agar, a tumorigenic phenotype, whereas four immortalized lines (ipn02.8, ipn97.4, ipNF03.3, and ipNF04.4) were able to form several colonies (but not nearly as many as the positive control malignant cells). These four lines also lost contact inhibition, another tumorigenic phenotype. Figure 5 shows an example of one of six cell lines that lost contact inhibition: as heterozygous cell line ipnNF95.11C became more confluent, it gained a more spindle-like morphology but continued to grow on top of the monolayer. But again reflecting heterogeneity, some immortalized lines did not lose contact inhibition, and one primary culture (pn02.8) never showed contact inhibition (unlike all others). Despite acquisition of tumorigenic properties in some of the cell lines, there was no evidence of microsatellite instability in any immortalized lines, or in primary cultured Schwann cells, suggesting that mismatch repair is intact in these cells (data not shown).

Cytogenetic analysis showed that while some of the cell lines had normal or near-normal karyotypes, some were aneuploid, including mosaic changes and near-tetraploidy (Supplementary Table S2). Increased Cdk4 expression affecting centrosome functioning is most likely responsible for the occasional occurrence of this latter phenotype. ${ }^{52}$ It is also possible that cytogenetic abnormalities may arise as random culture artifacts. For example, ipNF05.5's karyotype (46,XY,t $(2 ; 3)$ (q23;p26) [18]/46 47,sl,+9[cp2]) showed two cells with a gain of chromosome 9 not found in the mixed clone culture; however, the translocation was present in all of the cells of both samples (and was not from the germline), suggesting an event that occurred before the cloning and predominated 

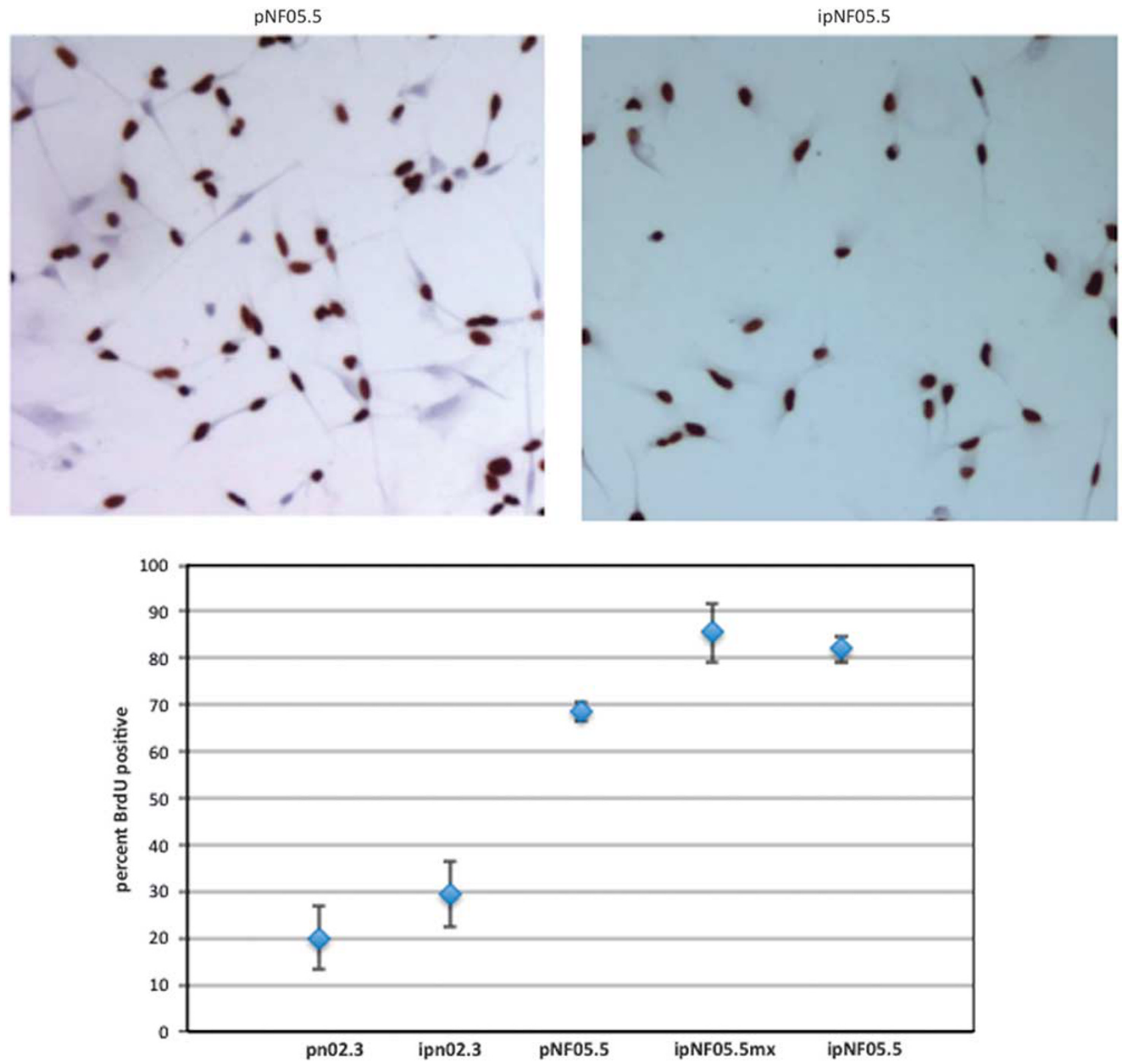

Figure 4 The top of the figure shows BrdU staining of proliferating cells in pNF05.5 and corresponding immortalized line ipNF05.5 and six-clone mix ipNF05.5mix. Fewer cells are shown in the immortalized photo, but a higher percentage of nuclei are BrdU-positive. The graph shows the proliferation indices (percentage of BrdU-positive cells) for these cells as well as normal SC pair pn02.3 and ipn02.3. Immortalized SC lines trended toward a higher proliferation index than the primary cultures but the differences were not statistically significant (paired $t$-test).

in culture by coincidence or possibly selection. Of the three primary cultures studied (pNF95.11b, pNF95.6, and pNF05.5), all had normal karyotype 46,XY, consistent with literature that suggests most neurofibroma-derived primary culture Schwann cells do not show somatic cytogenetic abnormalities, indicating that the karyotype abnormalities are strictly immortalization- and/or long-term culturerelated. ${ }^{32,53}$ There were no obvious relationships between the properties in Table 1 and aneuploidy; for example ipNF95.11b $\mathrm{C} / \mathrm{T}$ (normal karyotype) lost contact inhibition relative to primary, while ipNF95.6 did not (most cells with normal karyotype).
Out of curiosity, during this project, six immortalized cell lines (Table 1) were tested in simple xenograft experiments ( 2 mice, 1 nerve each) for the ability to form a visible intraneural (orthotopic) or subcutaneous tumor in immunocompromised mice (NOD.Cg-Prkdcscid Il2rgtm 1 Wjll/SzJ, Jackson Labs \#005557) with a two-month incubation (standard for our laboratory $\left.{ }^{16,39}\right)$. In our experience, Schwann-enriched primary normal nerve and plexiform SC cultures do not proliferate in the subcutaneous environment, but can survive and proliferate to a minor degree in the sciatic nerve, although not always producing outright visible enlargement of the sciatic nerve. ${ }^{16,39}$ None of the immortalized cell lines 

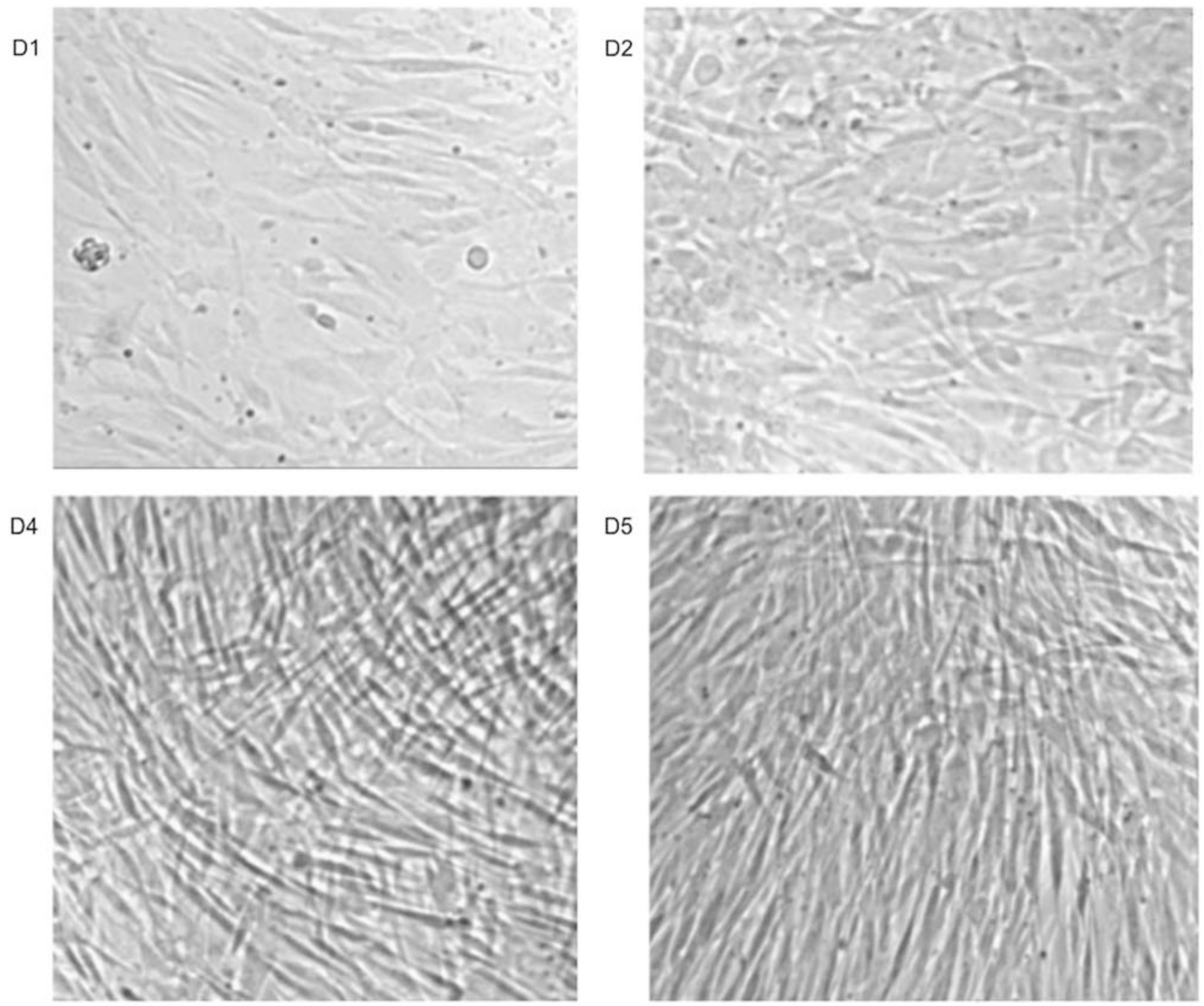

Figure 5 Phase contrast photographs of ipnNF95.11C cells at 1 day (D1), 2 days (D2), 4 days (D4), and 5 days after plating (D5). The cells showed lack of contact inhibition and grew over each other.

tested formed a detectable subcutaneous mass. In the sciatic nerve environment, lines ipNF06.2A and ipn02.3 formed no visible tumors. ipNF95.6 cells formed a very small intraneural tumor (Figure 6) consistent with primary cells previously published, ${ }^{16}$ and ipNF00.6 cells had a similar result. In contrast, ipNF04.4 (Figure 6) and ipNF03.3 formed massive intraneural tumors. Consistent with this observation, these latter two cell lines had some of the most tumorigenic phenotypes in vitro (eg, moderate anchorage independence, lack of contact inhibition), and proliferation rates in the immortalized lines were substantially higher than the respective primary cultures. These results are reminiscent of our previous NF1 sciatic xenograft results where one MPNST culture produced a small tumor while another produced a very large mass similar to that seen in ipNF03.3 and ipNF04.4. ${ }^{40,41}$ The transduction protocol for all primary cells was identical including the same vector preparations, so the end result in terms of tumorigenic phenotypes from this immortalization method is clearly somewhat unpredictable.

\section{Discussion}

This work shows that human Schwann cells, including those from neurofibromas, can be immortalized by exogenous expression of $C d k 4$ and telomerase. This addresses a need in the biomedical research community both by indicating a way to create immortalized human Schwann cells, and by creating these characterized cells as research resources. The advantages of the immortalized cells compared with primary cultures include increased purity, much greater expansion capacity, and reduced need for special culture conditions. The trade-off is that the cells have gained the tumorigenic property of immortalization, with variable additional transformed phenotypes such as anchorage independence (in four lines, although the degree of colony formation was much less than the positive 

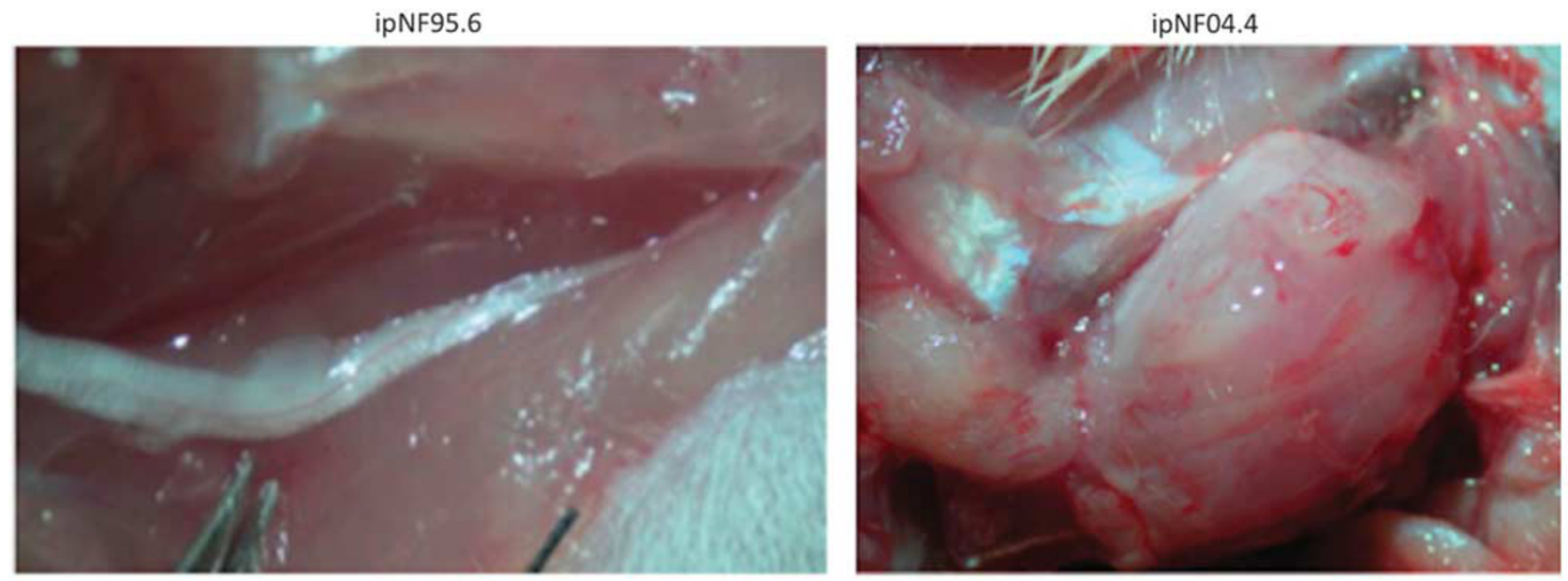

Figure 6 In situ photographs of tumors (2 month post-xenograft) of ipNF95.6 and ipNF04.4 cells into sciatic nerves of immunocompromised mice. ipNF95.6 only formed a small tumor/nerve enlargement (seen at the tip of forceps), whereas ipNF04.4 yielded a very large intraneural tumor. Similar, large intraneural tumors also resulted from cell line ipNF03.3 (not shown).

control, glioblastoma line T98G). We also observed that the process led to aneuploidy in some lines, which is unpredictable in its occurrence and effect, but immortalization with $C d k 4$ likely sets up a susceptibility for such events by affecting cell cycle. An established phenomenon in neurofibroma-derived two-hit enriched SC cultures is atypical morphology and variable staining with $\mathrm{S} 100 \mathrm{~B},{ }^{16}$ and this was also observed with some of the immortalized cell lines. Not unexpectedly, there was heterogeneity for nearly all features measured in the immortalized cells, with little in the way of patterns (eg, some lost contact inhibition and anchorage dependence, while others had only one or none of these features, and which did not correlate with presence of aneuploidy or other properties, or being wild-type, heterozygous or homozygous mutant at NF1). For four neurofibroma-derived SC lines (ipNF95.11b, ipNF95.6, ipNF05.5, and ipNF04.4), genetics showed that that regardless of morphology and S100B expression, these lines are derived from the original two-hit SC. Although a normal somatic allele was not observed, we cannot rule out presence of underlying immortalized fibroblasts or heterozygous SC (estimating $<5 \%$ if present). The ability to isolate single-cell clones enables study of such. On the other hand, study of a clone might yield results less representative of the tumor SC line as a whole.

This work is also the first to produce immortalized SC line pairs from normal nerve and plexiform neurofibroma from the same patients, which may be of utility in experiments where isogenic background is helpful: ipNF95.11b (C and C/T) with ipnNF95.11C; ipNF05.5 with ipnNF09.4.

Because they are purer than primary cells, and can expand many more passages, these immortalized SC cell lines can be useful for basic biology/neuroscience investigations (wildtype, heterozygous), tumor investigations (NF1-null cells), genetic manipulation to create new models or for rescue experiments, or preclinical studies such as use in xenograft as we demonstrated. For example, these cells were recently used in a high-throughput drug library, ${ }^{47}$ and in one study the wild-type ipn02.3 cell line was modified to knockdown the NF2 gene by siRNA lentivirus transduction. ${ }^{46}$ The availability of additional wild-type immortalized SC from this work (ipn02.8, ipn97.4) increases resources for such studies. Production of cell lines from different patients and tumors such as accomplished here also helps represent the heterogeneity between people and between neurofibromas, which may be very important in studies of NF1 pathogeneisis and therapeutics.

Supplementary Information accompanies the paper on the Laboratory Investigation website (http://www.laboratoryinvestigation.org)

\section{ACKNOWLEDGMENTS}

We thank the patients and their physicians who contributed the original samples. We gratefully acknowledge support in the form of a subcontract from Dr Karen Stephens (PI of DOD DAMD17-03-1-0203), as well as funding from the Neurofibromatosis Therapeutic Acceleration Program (NTAP; through Johns Hopkins University), and the Children's Tumor Foundation. We also appreciate insightful discussion and contributions from NTAP colleagues Marigo Stathis, Dannielle Ryman, Dr Jaishri Blakely, contribution of the retroviral vectors from Dr Woodring Wright (University of Texas Southwestern), and helpful discussions with Dr Anthony Yachnis, Dr Nancy Ratner, and Dr Annette Bakker. DOD DAMD17-03-1-0203 (NF Research Program), the Children's Tumor Foundation, Neurofibromatosis Therapeutic Acceleration Program.

\section{DISCLOSURE/CONFLICT OF INTEREST}

The authors declare no conflict of interest.

1. Evans DG, Howard E, Giblin C, et al. Birth incidence and prevalence of tumor-prone syndromes: estimate from a UK family genetic register service. Am J Med Genet A 2010;152A:327-332.

2. Karajannis MA, Ferner RE. Neurofibromatosis-related tumors: emerging biology and therapies. Curr Opin Pediatr 2015;27:26-33.

3. Lin AL, Gutmann DH. Advances in the treatment of neurofibromatosisassociated tumours. Nat Rev Clin Oncol 2013;10:616-624.

4. Colman SD, Williams CA, Wallace MR. Benign neurofibromas in type 1 neurofibromatosis (NF1) show somatic deletions of the NF1 gene. Nat Genet 1995;11:90-92. 
5. Kluwe L, Friedrich R, Mautner VF. Loss of NF1 allele in Schwann cells but not fibroblasts derived from an NF1-associated neurofibroma. Genes Chromosomes Cancer 1999;24:283-285.

6. Messiaen LM, Callens T, Mortier G, et al. Exhaustive mutation analysis of the NF1 gene allows identification of $95 \%$ of mutations and reveals a high frequency of unusual splicing defects. Hum Mutat 2000;15:541-555.

7. Anastasaki C, Woo AS, Messiaen LM, et al. Elucidating the impact of neurofibromatosis-1 germline mutations on neurofibromin function and dopamine-based learning. Hum Mol Genet 2015;24:3518-3528.

8. Laycock-van Spyk S, Thomas N, Cooper DN, et al. Neurofibromatosis type 1-associated tumours: their somatic mutational spectrum and pathogenesis. Hum Genomics 2011;5:623-690.

9. Ratner N, Miller SJ. A RASopathy gene commonly mutated in cancer: the neurofibromatosis tumour suppressor gene. Nat Rev Cancer 2015;15:290-301.

10. Hirbe AC, Dahiya S, Miller CA, et al. Whole exome sequencing reveals the order of genetic changes during malignant transformation and metastasis in a single patient with NF1-plexiform neurofibroma. Clin Cancer Res 2015;21:4201-4211.

11. Emmerich D, Zemojtel T, Hecht J, et al. Somatic neurofibromatosis type 1 (NF1) inactivation events in cutaneous neurofibromas of a single NF1 patient. Eur J Hum Genet 2015;23:870-873.

12. Spurlock G, Knight SJ, Thomas N, et al. Molecular evolution of a neurofibroma to malignant peripheral nerve sheath tumor (MPNST) in an NF1 patient: correlation between histopathological, clinical and molecular findings. J Cancer Res Clin Oncol 2010;136:1869-1880.

13. Pemov A, Li H, Patidar R, et al. Exome sequencing of neurofibromatosis type 1-associated plexiform neurofibromas reveals the primacy of NF1 loss as the driver of tumorigenesis. In revision.

14. Serra E, Rosenbaum T, Winner $U$, et al. Schwann cells harbor the somatic NF1 mutation in neurofibromas: evidence of two different Schwann cell subpopulations. Hum Mol Genet 2000;9:3055-3064.

15. Rosenbaum T, Rosenbaum $C$, Winner $U$, et al. Long-term culture and characterization of human neurofibroma-derived Schwann cells. J Neurosci Res 2000;61:532-564.

16. Muir D, Neubauer D, Lim IT, et al. Tumorigenic properties of neurofibroma Schwann cells. Am J Pathol 2001;58:501-513.

17. Rubin H. Cell aging in vivo and in vitro. Mech Ageing Dev 1997;98:1-35.

18. Pagano JS, Jiminez G, Sung NS, et al. Epstein-Barr viral latency and cell immortalization as targets for antisense oligomer. Ann N Y Acad Sci 1992;660:107-118.

19. Stoner GD, Kaighn ME, Reddel RR, et al. Establishment and characterization of SV40 T antigen immortalized human esophageal epithelial cells. Cancer Res 1991;51:365-371.

20. Douglas JF, Quinlan MP. Efficient nuclear localization and immortalizing ability, two functions dependent on the adenovirus type 5 (Ad5) E1A second exon, are necessary for cotransformation with Ad5 E1B but not with T24ras. J Virol 1995;69:8061-8065.

21. Hawley-Nelson P, Vousden KH, Hubbert NL, et al. HPV16 E6 and E7 proteins cooperate to immortalize human foreskin keratinocytes. Embo J 1989:8:3905-3910.

22. Lehmann HC, Chen W, Mi R, et al. Human Schwann cells retain essential phenotype characteristics after immortalization. Stem Cells Dev 2012;21:423-431.

23. Bodnar $A G$, Ouelette $M$, Frolkis $M$, et al. Extension of life span by introduction of telomerase into normal human cells. Science 1998;279:349-352.

24. Yang J, Chang E, Cherry AM, et al. Human endothelial cell life extension by telomerase expression. J Biol Chem 1999;274:26141-26148.

25. Dickson MA, Hahn WC, Ino Y, et al. Human keratinocytes that express hTERT and also bypass p16(INK4a)-enforced mechanism that limits life span become immortal yet retain normal growth and differentiation characteristics. Mol Cell Biol 2000;20:1436-1447.

26. Foster SA, Galloway DA. Human papillomavirus type 16 E7 alleviates a proliferation block in early passage mammary epithelial cells. Oncogene 1996;12:1773-1779.

27. Brenner AJ, Stampfer MR, Aldaz CM. Increased p16 expression with first senescence arrest in human mammary epithelial cells and extended growth capacity with p16 inactivation. Oncogene 1998;17:199-205.

28. Puthenveettil JA, Burger MS, Reznikoff CA. Replicative senescence in human uroepithelial cells. Adv Exp Med Biol 1999;462:83-91.

29. Darbro BW, Lee KM, Nguyen NK, et al. Methylation of the p16(INK4a) promoter region in telomerase immortalized human keratinocytes co-cultured with feeder cells. Oncogene 2006;25:7421-7433.
30. Ramirez RD, Sheridan S, Girard L, et al. Immortalization of human bronchial epithelial cells in the absence of viral oncoproteins. Cancer Res 2004;64:9027-9034.

31. Gutmann DH, Aylsworth A, Carey JC, et al. The diagnostic evaluation and multidisciplinary management of neurofibromatosis 1 and neurofibromatosis 2. JAMA 1997;278:51-57.

32. Wallace MR, Rasmussen SA, Lim IT, et al. Culture of cytogenetically abnormal Schwann cells from benign and malignant NF1 tumors. Genes Chromosomes Cancer 2000;27:117-123.

33. Chang $\sqcup$. Lentiviral vector transduction of dendritic cells for novel vaccine strategies. Methods Mol Bio 2010;614:161-170.

34. Oka M, Chang LJ, Costatini F, Terada N. Lentiviral vector-mediated gene transfer in embryonic stem cells. Methods Mol Biol 2006;329: 273-281.

35. Techangamsuwan S, Kreutzer R, Kreutzer M, et al. Transfection of adult canine Schwann cells and olfactory ensheathing cells at early and late passage with human TERT differentially affects growth factor responsiveness and in vitro growth. J Neurosci Methods 2009;176:112-120.

36. Abernathy CR, Rasmussen SA, Stalker HJ, et al. NF1 mutation analysis using a combined heteroduplex/SSCP approach. Hum Mutation 1997;9:548-554.

37. Thomson SAM, Wallace MR. RT-PCR splicing analysis of the NF1 open reading frame. Hum Genet 2002;110:495-502.

38. Boland CR, Thibodeau SN, Hamilton SR, et al. A National Cancer Institute Workshop on Microsatellite instability for cancer detection and familial predisposition: development of international criteria for the determination of microsatellite instability in colorectal cancer. Cancer Res 1998;58:5248-5257.

39. Li H, Zhang L, Fishbein L, et al. Analysis of steroid hormone effects on xenografted human NF1 tumor Schwann cells. Cancer Biol Ther 2010;10:758-764.

40. Perrin GQ, Li H, Fishbein L, et al. An orthotopic xenograft model of intraneural NF1 MPNST suggests a potential association between steroid hormones and tumor cell proliferation. Lab Invest 2007a;87: 1092-1102.

41. Perrin GQ, Fishbein $L$, Thomson SA, et al. Plexiform-like neurofibromas develop in the mouse by intraneural xenograft of an NF1 tumorderived schwann cell line. J Neurosci Res 2007b;85:1347-1357.

42. Rahrmann EP, Moriarty BS, Otto GM, et al. Trp53 haploinsufficiency modifies EGFR-driven peripheral nerve sheath tumorigenesis. Am J Pathol 2014;184:2082-2098.

43. Watson AL, Rahrmann EP, Moriarity BS, et al. Canonical Wnt/ $\beta$-catenin signaling drives human Schwann cell transformation, progression, and tumor maintenance. Cancer Discov 2013;3:674-689.

44. Rahrmann EP, Watson AL, Keng WW, et al. Forward genetic screen for malignant peripheral nerve sheath tumor formation identifies novel genes and genetic pathways driving tumorigenesis. Nat Genet 2013;45:756-766.

45. Keng $\mathrm{V}$, Watson $\mathrm{AL}$, Rahrmann $\mathrm{EP}$, et al. Conditional inactivation of Pten with EGFR overexpression in Schwann cells models sporadic MPNST. Sarcoma 2012;72:3405-3413.

46. James M, Stivison E, Beauchamp R, et al. Regulation of mammalian target of rapamycing complex 2 (mTORC2) signaling in NF2-deficient target cell types. Mol Cancer Res 2012;10:649-659.

47. Ryman DA, Wallace MR, Thomas C, et al. A 1536-Well Quantitative High-Throughput Screen to Identify Therapeutic Compounds Targeting Plexiform Neurofibromas Tumor Cells. In preparation.

48. Uchida T, Matozaki T, Suzuki T, et al. Expression of two types of neurofibromatosis type 1 gene transcripts in gastric cancers and comparison of GAP activities. Biochem Biophys Res Commun 1992;187:332-339.

49. Barron VA, Lou $\mathrm{H}$. Alternative splicing of the neurofibromatosis type 1 pre-mRNA. Biosci Rep 2012;32:131-138.

50. Gutmann DH, Geist RT, Wright DE, et al. Expression of the neurofibromatosis 1 (NF1) isoforms in developing and adult rat tissues. Cell Growth Differ 1995;6:315-323.

51. Loda-Hutchinson R. Analysis of NF1 Mutation Mechanisms. PhD Dissertation, University of Florida, Gainesville, FL, 2009.

52. Adon AM, Zeng $\mathrm{X}$, Harrison MK, et al. Cdk2 and Cdk4 regulate the centrosome cycle and are mediators of centrosome amplification in p53-null cells. Mol Cell Biol 2010;30:694-710.

53. Mertens F, Dal Cin P, De Wever I, et al. Cytogenetic characterization of peripheral nerve sheath tumours: a report of the CHAMP study group. J Pathol 2000;190:31-38. 